\title{
Las TIC y su contribución en la administración de la producción de la PYMES en la Provincia de Huaura
}

\author{
The tics and their contribution in the administration of the production of SMES \\ in the province of Huaura
}

Manuel Alberto Patroni Bazalar ${ }^{1}$, Miguel Ángel Luna Neyra', Daniel Alberto Valenzuela Narvaez ${ }^{1}$

\begin{abstract}
RESUMEN
Objetivo: Determinar en qué medida las TIC contribuyen a la administración a la producción de las PYMES de la Provincia de Huaura. Materiales y Métodos: Método hipotético-deductivo, cuyo diseño es el no experimental transversal correlacional. Así mismo la población a investigar está determinada por 5,199 MYPES de la Provincia de Huaura, con una muestra de 357 empresas de este tipo, se aplicando el sistema de encuestas por cuestionario mediante el sistema de muestreo aleatorio simple. Resultados: Se obtuvo como resultado que un 97.5\% de las PYMES si cuentan con internet en sus negocios y un $2.5 \%$ no cuentan, por otro lado un $77.03 \%$ de consideran que las TIC a mejorado su proceso de producción, un $79.83 \%$ de los empresarios consideran que las TIC han dado mucho mayor acceso a sus mercados, mientras que un $16.53 \%$ de los empresarios han manifestados que regularmente le han dado acceso a su mercados, así también un $88.24 \%$ de los empresarios consideran que las TIC han contribuido al mejoramiento de la calidad y diseño de sus productos y un $9.80 \%$ consideran regularmente, y un $96.64 \%$ de los empresarios manifestaron que las TIC si han influenciado en el cambio de estrategia de su sistema de producción y un 3.36\% dijeron que no. Conclusión: Las TIC si contribuyen en la administración de la PYMES en la Provincia de Huaura.
\end{abstract}

Palabras claves: Administración, producción, productividad, adopción, competitividad

\begin{abstract}
Objective: Determine in what measure the TIC contributes and the administration of the production of the PYMES of the County of Huaura. The used Materials the Method is determined by the hypothetical-deductive Method whose design is not the experimental traverse correlacional. Likewise the population to investigate is determined by 5,199 MYPES of the County of Huaura, with a sample of 357 companies of this type, you applying the system of surveys for questionnaire by means of the system of simple random sampling. Results: it was obtained as a result that $97.5 \%$ of the PYMES if they have internet in their business and $2.5 \%$ they don't count, on the other hand $77.03 \%$ of they consider that the TIC had improved their production process, $79.83 \%$ of the managers considers that the TIC has given much bigger access to its markets, while $16.53 \%$ of the managers has manifested that regularly they have given access to its markets, likewise an or the managers' $88.24 \%$ consider that the Tic has contributed to the improvement of the quality and design of their products and $9.80 \%$ they consider regularly, and $96.64 \%$ of the managers manifested that the TIC if they have influenced in the change of strategy of its production system and $3.36 \%$ they said that $\mathrm{nr}$. Conclusions; the Tic if they contribute in the administration of the PYMES in the County of Huaura.
\end{abstract}

Key words: Administration, production, productivity, adoption, competitiveness 


\section{INTRODUCCIÓN}

En las condiciones actuales de globalización de los mercados, las organizaciones como sistemas tienen la necesidad de aumentar su competitividad en términos de crecimiento y desarrollo donde la innovación y la administración de la tecnología son consideradas variables motrices del sistema. La administración de la tecnología demanda de modelos y metodologías que sean aplicables al entorno específico de los sistemas. En el presente trabajo se propone un modelo de tres vectores para identificar al insumo tecnológico en las organizaciones desde un punto de vista sistémico y una metodología para su administración con un enfoque estratégico y pragmático. Es así que una forma de promover el desarrollo, reforzamiento y mejora de la competitividad de las PYMES en un país, es a través de la creación de condiciones que permitan a estas empresas participar en el flujo internacional del comercio. Sin embargo, para crear las condiciones adecuadas para su participación en el contexto actual de una economía mundial globalizada y automatizada, es fundamental que las PYMES estén preparadas para trabajar con los nuevos niveles tecnológicos, para poder adoptar los nuevos flujos de información y comunicaciones.

Como se observar la Provincia de Huaura durante estos últimos años su población ha venido acrecentándose debido a que esta es la capital de la Región lo que motiva a una migración constante de las zonas de influencia de nuestro Región generando desocupación, pobreza. Sin embargo hoy en día se constituyen en una de las provincias más prosperas del norte chico ya que cuentan con un buen número de MYPES que se constituyen en el soporte socioeconómico de esta parte de nuestro país. Por esta razón nos parece muy importante tocar uno de los temas de gran importancia para el desarrollo de nuestra provincia, que lo hemos titulado: "LAS TIC Y SU CONTRIBUCIÓN EN LA ADMINISTRACIÓN DE LA PRODUCCIÓN DE LA PYMES EN LA PROVINCIA DE HUAURA".

En tal sentido nos hemos planteado la siguiente hipótesis: "Las TIC contribuyen en la administración de la producción de las PYMES de la Provincia de Huaura" esta nos ha permitido desarrollar de una amanera fehaciente los problemas planteados y así alcanzar el objetivo fundamental como determinar en qué medida las TIC contribuyen en la administración de la producción de las PYMES de la Provincia de Huaura.

Lo que hemos podido demostrado que las TIC se han convertido en una de las técnicas más aplicadas en los sistemas administrativos en este tipo de organizaciones y por ende en los procesos de producción de toda empresa tanto para la producción de productos bienes como servicios. Es así que las TIC permiten alcanzar una mayor calidad en los productos y una evidente mayor producción y productividad de las empresas y sobre todo en las MYPES de nuestra Provincia de Huaura. Se dice que empresas que no se adapten a las nuevas tecnologías, especialmente que no formen parte de empresas redes, simplemente dejaran de existir, puesto que estas no tendrán las herramientas necesarias para poder competir.

\section{MATERIALY MÉTODOS}

\section{Diseño Metodológico}

El diseño metodológico es no experimental transversal correlacional.

\section{Población}

La población de la presente investigación está determinada por el total de las PYMES de la Provincia de Huaura tal como mostraremos en el cuadro, que está determinado por una población de 5,199 empresas de acuerdo a la cámara de Comercio y producción de la Provincia de Huaura.

\section{Muestra}

Considerando que de los doce distritos todos no son relevantes para poder llevar a cabo el estudio de investigación hemos creído pertinente considerar a los distritos de Huacho, Hualmay, Santa María y Carquin y Vegueta para determinar nuestro universo para la presente investigación.

Tabla 1: Distritos con sus Unidades Económicas.

\begin{tabular}{clc}
\hline $\mathbf{N}^{\circ}$ & DISTRITOS & UNIDADES ECONÓMICAS \\
\hline $\mathbf{1}$ & Carquin & 102 \\
$\mathbf{2}$ & Huacho & 3,406 \\
$\mathbf{3}$ & Hualmay & 333 \\
$\mathbf{4}$ & Santa María & 1,013 \\
$\mathbf{5}$ & Vegueta & 345 \\
\hline & TOTAL & $\mathbf{5 , 1 9 9}$ \\
\hline
\end{tabular}

En principio se ha determinado el Muestreo Aleatorio simple debido a que existen las condiciones que garantizan que la distribución de $p$ es aproximadamente normal .Por tanto se obtiene la fórmula para $\mathbf{n}$, ya que es lo suficiente grande como para hacer innecesario uso de la correlación finita, por cuanto se ha considerado la formula siguiente :

$$
\begin{aligned}
& n=\frac{Z^{2} p q N}{E^{2}(N-1)+Z^{2} p q} \\
& Z=(1,96)^{2} \\
& \mathrm{P}=\text { Proporción de población de interés. } \\
& q=(1-p) \\
& N=\text { Universo } \\
& E=(0.05)^{2} \\
& \mathrm{n}=\text { Tamaño de la muestra } \\
& \begin{array}{c}
n=\frac{(1.96)^{2}(0,5)(0,5)(5,199)}{(0,05)^{2}(5,199-1)+(1.96)^{2}(0,5)(0,5)} \\
n=-4,993 \\
-14
\end{array}
\end{aligned}
$$

Enero - Marzo 2017 
Tabla 2: Para llevar a cabo las encuestas en las unidades económicas.

\begin{tabular}{llcc}
\hline $\mathbf{N}^{\circ}$ & DISTRITOS & $\begin{array}{c}\text { UNIDADES } \\
\text { ECONÓMICAS }\end{array}$ & $\begin{array}{c}\mathbf{N}^{\circ} \mathbf{D E} \\
\text { PYMES }\end{array}$ \\
\hline $\mathbf{1}$ & Carquin & 102 & 07 \\
$\mathbf{2}$ & Huacho & 3,406 & 233 \\
$\mathbf{3}$ & Hualmay & 333 & 23 \\
$\mathbf{4}$ & Santa María & 1,013 & 70 \\
$\mathbf{5}$ & Vegueta & 345 & 24 \\
\hline & TOTAL & $\mathbf{5 , 1 9 9}$ & $\mathbf{3 5 7}$ \\
\hline
\end{tabular}

En etapa de recolección de datos de la investigación se conoce también como trabajo de campo. Estos datos o información que va a recolectarse son el medio a través del cual: Probaremos la hipótesis. Responderemos las preguntas de investigación. Y lograremos los objetivos del estudio originados del problema de investigación. Los datos los obtendremos de:

Fuentes: Primaria (Personas y Hechos) y Secundaria (Material Impreso).

Técnicas Principales: Tenemos la encuesta (Cuestionario), Entrevista (Personal), Observación (Personal Directa y Medios electrónicos) e Internet.

Se utilizó los estadísticos siguientes: estadísticos de tendencia central media, mediana, estadísticos de dispersión, desviación estándar, varianza, tabla de frecuencias, razones y proporciones.

\section{RESULTADOS}

Tabla 3: Porcentaje de negocios que cuentan con internet en la Provincia de Huaura

\begin{tabular}{lccccccc}
\hline \multicolumn{1}{c}{ Carquin } & Huacho & Huaura & Santa Maria & Vegueta & Sub Total $\%$ \\
\hline SI & 06 & 230 & 21 & 68 & 23 & 348 & 97.5 \\
NO & 01 & 03 & 02 & 02 & 01 & 09 & 2.5 \\
\hline TOTAL & $\mathbf{0 7}$ & $\mathbf{2 3 3}$ & $\mathbf{2 3}$ & $\mathbf{7 0}$ & $\mathbf{2 4}$ & $\mathbf{3 5 7}$ & 100 \\
\hline
\end{tabular}

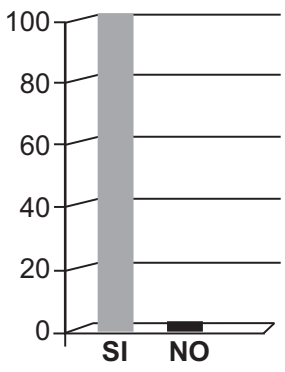

Figura 1: De acuerdo a la gráfica un $97.5 \%$ de las PYMES de nuestra Provincia de Huaura manifestaron que si cuentan con internet en sus negocios y un $2.5 \%$ no cuentan de lo cual podemos inferir que la gran mayoría de estas empresas consideran necesaria e imprescindible contar con internet en las actividades empresariales.
Tabla 4: Mejoramiento de la producción en función a la relación con las TIC.

\begin{tabular}{|c|c|c|c|c|c|c|c|}
\hline & Carquin & Huacho & Hualmay & Santa María & Vegueta & $\begin{array}{l}\text { Sub- } \\
\text { Total }\end{array}$ & $\%$ \\
\hline Mucho & 05 & 93 & 13 & 19 & 19 & 275 & 77.03 \\
\hline Regular & 01 & 35 & 07 & 03 & 69 & 69 & 19.33 \\
\hline Poco & 00 & 05 & 03 & 01 & 11 & 11 & 3.08 \\
\hline Nada & 01 & 00 & 00 & 01 & 02 & 02 & 0.56 \\
\hline TOTAL & 07 & 233 & 70 & 24 & 357 & 357 & 100 \\
\hline
\end{tabular}

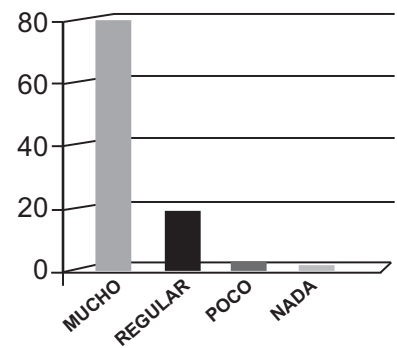

Figura 2: Como podemos observar un $77.03 \%$ de las MYPES de nuestra Provincia de Huaura consideran que las TIC a mejorado mucho en su proceso de producción, mientras que un 19,33\% considera que ha mejorado regularmente en su proceso de producción, sin embargo hay un porcentaje muy bajo en cuanto a poco o nada, como es evidente una vez más las TIC son consideradas hoy en día como parte determinante en sus actividades no solo en su proceso de producción sino también en la administración de la empresa en todas las actividades operativas.

Tabla 5: Las TIC y su mayor acceso de la MYPES a sus mercados.

\begin{tabular}{|c|c|c|c|c|c|c|c|}
\hline & Carquin & Huacho & Hualmay & Santa María & Vegueta & $\begin{array}{l}\text { Sub- } \\
\text { Total }\end{array}$ & $\%$ \\
\hline Mucho & 04 & 196 & 15 & 55 & 15 & 285 & 79.8 \\
\hline Regular & 02 & 34 & 05 & 12 & 06 & 59 & 16.5 \\
\hline Poco & 00 & 03 & 02 & 03 & 02 & 10 & 2.9 \\
\hline Nada & 01 & 00 & 01 & 00 & 01 & 3 & 0.8 \\
\hline TOTAL & 07 & 233 & 23 & 70 & 24 & 357 & 100 \\
\hline
\end{tabular}

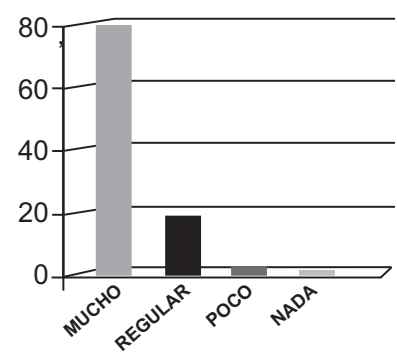

Figura 3: De conformidad a la grafica el $79.83 \%$ de los empresarios de las PYMES de nuestra provincia de Huaura consideran que las TIC han dado mucho 
mayor acceso a sus mercados , mientras que un 16.53 $\%$ de los empresarios han manifestados que regularmente le han dado acceso a su mercados, lo cual nos permite inferir que las TIC son tan importante en la comercialización de sus productos ya que estos sistemas permite ampliar nuevos mercados mediante las redes sociales que hoy en día son parte de las estrategias de toda empresa por más pequeña que esta sea y más aún en una pequeña empresa, que desde su constitución ya cuentan con una computadora y por ende con internet.

Tabla 6: Contribución de las TIC en la calidad de los productos.

\begin{tabular}{lccccccc}
\hline \multicolumn{2}{c}{ Carquin Huacho Hualmay Santa María } & Vegueta & $\begin{array}{c}\text { Sub- } \\
\text { Total }\end{array}$ & $\%$ \\
\hline Mucho & 04 & 219 & 16 & 60 & 18 & 315 & 88.24 \\
Regular & 02 & 11 & 06 & 10 & 06 & 35 & 9.80 \\
Poco & 01 & 03 & 01 & 00 & 00 & 05 & 1.40 \\
Nada & 00 & 00 & 00 & 00 & 00 & 00 & 0.00 \\
\hline TOTAL & $\mathbf{0 7}$ & $\mathbf{2 3 3}$ & $\mathbf{2 3}$ & $\mathbf{7 0}$ & $\mathbf{2 4}$ & $\mathbf{3 5 7}$ & $\mathbf{1 0 0}$ \\
\hline
\end{tabular}

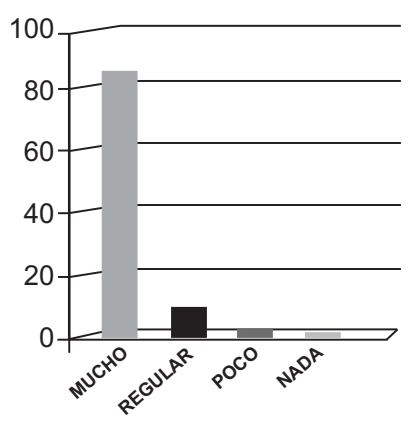

Figura 4: De acuerdo a los resultados obtenidos en la tabla y grafico correspondiente un $88.24 \%$ de los empresarios consideran que las Tic han contribuido mucho en el mejoramiento de la calidad de sus productos y un $9.80 \%$ consideran regularmente, como es evidente la calidad de todo producto está en función directo a la tecnología basado en los software que actualmente se bien aplicando en el diseño de productos (AUTOCAD) sistema computarizados en los procesos producción, así como en la administración en general de toda empresa y por ende en las MYPES de nuestra provincia de Huaura.

Tabla 7: Contribución de las TIC en la gestión de sus insumos.

\begin{tabular}{|c|c|c|c|c|c|c|c|}
\hline & Carquin & Huacho & Hualmay & Santa María & Vegueta & $\begin{array}{l}\text { Sub- } \\
\text { Total }\end{array}$ & $\%$ \\
\hline Mucho & 03 & 213 & 18 & 60 & 18 & 312 & 89.9 \\
\hline Regular & 02 & 17 & 05 & 08 & 06 & 38 & 10.6 \\
\hline Poco & 02 & 03 & 00 & 02 & 00 & 07 & 1.95 \\
\hline Nada & 00 & 00 & 00 & 00 & 00 & 00 & 0.00 \\
\hline TOTAL & 07 & 233 & 23 & 70 & 24 & 357 & 100 \\
\hline
\end{tabular}

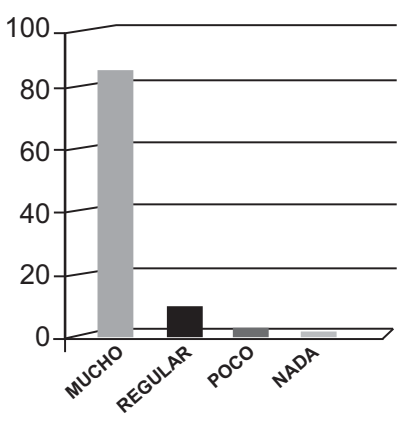

Figura 5: De conformidad a los resultados obtenidos en la tabla y gráfica correspondiente se puede observar que un $89.91 \%$ de los empresarios consideran que las TIC han determinado una mayor eficiencia en el uso y gestión de sus insumos y un 10.63 considera una regular eficiencia , ante esta situación se puede inferir que las TIC bien siendo una herramienta fundamental para poder adquirir sus insumos,componentes y otros materiales para el proceso de producción como para su uso mediante la aplicación de una buena administración de sus materiales, mediante la aplicación de algunos programas como el Software ERP que sirve para la gestión de empresas de transporte de mercancía y logística, gestión de tráfico, logística y almacenaje, MRP I, (Enterprise Resource Planning) o sistemas de planeamiento de recursos de la empresa, es una herramienta de estrategia, que equipa a la empresa con las capacidades y recursos necesarios para integrar y sincronizar las funciones asiladas en un proceso continuo de negocios.

Tabla 8: Las TIC y su contribución en el sistema de producción de las PYMES.

\begin{tabular}{|c|c|c|c|c|c|c|c|}
\hline & Carquin & Huacho & Hualmay & Santa María & Vegueta & $\begin{array}{l}\text { Sub- } \\
\text { Total }\end{array}$ & $\%$ \\
\hline SI & 04 & 228 & 22 & 68 & 23 & 345 & 96.6 \\
\hline NO & 03 & 05 & 01 & 02 & 01 & 12 & 3.4 \\
\hline TOTAL & 07 & 233 & 23 & 70 & 24 & 357 & 100 \\
\hline
\end{tabular}

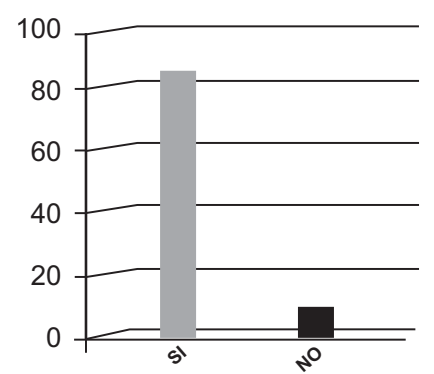

Figura 6: En consideración a los resultados obtenido un $96.64 \%$ de los empresarios manifestaron que las TIC si han influenciado en el cambio de estrategia de su sistema de producción y un $3.36 \%$ dijeron que no. Ante estos resultados podemos decir que hoy en día las tic han revolucionado la administración de los sistemas de producción desarrollando de esta manera sus capacidades de producción, logística, 
financiera, comercial y personal mediante la aplicación del E-Procurement cuya función es la automatización de los procesos interno y externos relacionados con el requerimiento de compras ,suministros, pago y control por medio de Internet como medio principal de comunicación clienteproveedor, así también en la gestión integral del proceso de producción como cero stock, plazo de entrega inmediato, producción bajo pedido, integración de producción-logística-finanzas, integración empresa proveedores y la integración de los factores humanos.

\section{DISCUSIÓN}

En el entorno competitivo actual caracterizado por la globalización de los mercados, lo que supone nuevas oportunidades de negocio y aumento de la competencia; por las nuevas formas de colaboración entre empresas PYMES; la búsqueda de agentes y funciones que generen valor añadido y, finalmente, la necesidad de adaptación de los procesos de negocio a los cada vez más rápidos cambios en el entorno, las Tecnologías de la Información y las Comunicaciones (TIC) desempeñan un papel decisivo, ofreciendo a las empresas la flexibilidad y el dinamismo necesarios para competir en este nuevo escenario.

La presentación de algunos estudios de TIC para PYMES han demostrado que es un hecho que las PYMES necesitan incorporar tecnología a sus estrategias de negocio para poder ser más productivas, aumentar su grado de eficiencia y así llevar a cabo una gran contribución para la economía de un País. Esa contribución a la economía del país, contrario a lo que se pudiera pensar, es de suma importancia. Se considera que más del $80 \%$ de los negocios son micro, pequeños y medianos en él Perú y concentran cerca del $50 \%$ de los ingresos del país. Ante esta información nos podemos dar cuenta de la importancia que tienen las PYMES en el nivel de macroeconómico de un país.

La Sociedad Actual se caracteriza por la utilización de la información para generar conocimiento, con el fin de mejorar los procesos de cualquier organización: la información es un bien cada vez menos restringido, más compartido y la ventaja competitiva de las organizaciones radica en interpretarla y convertirla en un elemento diferencial, en un activo productivo y rentable. Los antiguos sistemas de información usados, que convertían datos operacionales en indicadores de gestión (la mayor parte de las veces de naturaleza económico-financiera), se han visto absorbidos y superados por un nuevo concepto del tratamiento de la información para la toma decisiones, evoluciona con fuerza en el ámbito de las Tecnologías de la Información.

Este cambio ha sido propiciado por la propia evolución de las TIC, que permiten un tratamiento cada vez más rápido, complejo e inmediato de los datos, la información y, en definitiva, el conocimiento. Una de las claves estratégicas que se va a valorar cada vez más es la capacidad de una empresa para dar Inteligencia a su Negocio a través de la Gestión y
Administración en la Economía Digital. Para pasar de los modelos tradicionales de negocio a modelos nuevos que sean sostenibles, es necesario experimentar. En este punto, expertos usan el término "Economía del Conocimiento" para describir la "Economía Digital". Estos experimentos, sin embargo no deben considerarse como golpes aislados, sino como acciones pioneras para la transformación del negocio actual en un negocio basado en Internet.

\section{CONCLUSIONES}

Como podemos observar de acuerdo a los resultados obtenidos el $97.5 \%$ de las PYMES de nuestra Provincia de Huaura manifestaron que si cuentan con internet en sus negocios y un $2.5 \%$ no, lo cual nos permite establecer que actualmente las TIC vienen alcanzado una gran relevancia en las actividades productivas de nuestra provincia de Huaura,

El proceso de producción es uno de los factores más importantes de toda empresa y por ende de las PYMES ya que estas deben estar a la vanguardia de los avances tecnológicos lo que permite establecer que de acuerdo a los resultados obtenidos un $77.03 \%$ de los empresarios de estas empresas consideraron que las TIC son parte muy importante de en el desarrollo de sus procesos de producción.

Como sabemos los mercados cada vez son más competitivos lo que hoy en día es una preocupación constante de los empresarios de todo tipo de empresas, en tal sentido de acuerdo al estudio realizado el $79.83 \%$ de los empresarios de las PYMES de nuestra provincia de Huaura consideran que las TIC han dado mucho mayor acceso a sus mercados, mientras que un $16.53 \%$ de los empresarios dijeron que no. Ante estos resultados es necesario puntualizar que si existe un gran interés por estas técnicas.

Los procesos de producción y la gestión de materiales son tan importantes como el planeamiento de toda actividad empresarial, lo cual determina la gran importancia que tienen las TIC hoy en día es tan determinante para que estas sobrevivan.

\section{AGRADECIMIENTOS}

Nuestro agradecimiento a los empresarios de las PYMES de la Provincia de Huaura por habernos dado la oportunidad de entrevistarlos y darnos los datos fehacientes que han permitido concluir esta investigación en forma objetiva y real. 


\section{REFERENCIAS BIBLIOGRÁFICAS}

AMIPICI. (2007). "Uso de nuevas Tecnologías Usuarios de Internet en México" Guadalajara_ México.

Anzola, S. (2003): Administración de pequeñas empresas. México: McGraw-Hill

Cabrera, Á. (2012). "Las Pymes: quienes son, como son y qué hacer con ellas" Universidad de Concepción. "La Paz-Bolivia

Casalet, M. y González, L. (2004) “Las tecnologías de la información en las pequeñas y medianas empresas mexicanas Scripta"Universidad de Barcelona- España.

CEPAL (2003) "Las Pymes en América latina y el Caribe: situación actual y desafíos"

Fernández, R (2005) “Las Pymes en México”

Galin, H. (1998). Guía para la Pequeña Empresa. Ediciones Genika, México.

IT PYMES (2011) "Las pymes deben aprovechar más el potencial de Internet para exportar" -Guadalajara-México.
Ríos, M. D. (2006). "El pequeño empresario en ALC las TIC y el Comercio Electrónico". Instituto para la Conectividad en las Américas-México.

TamezA.(2007) "Las pequeñas y medianas empresas y las tecnologías de información" explicado en Gestiopolis -México

Taszma, P. (2011) "Control de la Información el Futuro de la Pymes" artículo explicado en GestioPolis -México

Tanembaum, A. S. (2003). "Redes Computacionales". Madrid: Prentice Hall International Edition.España.

Zorrilla S. , Juan P. (2004) "La importancia de las Pymes en México y para el mundo" GestiopolisMéxico: 